\title{
Network bursts in cortical cultures are best simulated using pacemaker neurons and adaptive synapses
}

\author{
T. A. Gritsun · J. Le Feber · J. Stegenga • \\ W. L. C. Rutten
}

Received: 22 October 2008 / Accepted: 25 January 2010 / Published online: 16 February 2010

(C) The Author(s) 2010. This article is published with open access at Springerlink.com

\begin{abstract}
One of the most specific and exhibited features in the electrical activity of dissociated cultured neural networks (NNs) is the phenomenon of synchronized bursts, whose profiles vary widely in shape, width and firing rate. On the way to understanding the organization and behavior of biological NNs, we reproduced those features with random connectivity network models with 5,000 neurons. While the common approach to induce bursting behavior in neuronal network models is noise injection, there is experimental evidence suggesting the existence of pacemaker-like neurons. In our simulations noise did evoke bursts, but with an unrealistically gentle rising slope. We show that a small subset of 'pacemaker' neurons can trigger bursts with a more realistic profile. We found that adding pacemaker-like neurons as well as adaptive synapses yield burst features (shape, width, and height of the main phase) in the same ranges as obtained experimentally. Finally, we demonstrate how changes in network connectivity, transmission delays, and excitatory fraction influence network burst features quantitatively.
\end{abstract}

Keywords Recurrent neural networks · Cultured neuronal networks $\cdot$ Pacemaker cells $\cdot$ Network bursts

The work presented in this article was funded by the EU Marie-Curie Neurovers-IT project (MRTN-CT-2005-019247).

T. A. Gritsun ( $\varangle)$ · J. Le Feber · J. Stegenga · W. L. C. Rutten Institute for Biomedical Technology and Technical Medicine (MIRA), University of Twente, P.O. Box 217,

7500 AE Enschede, The Netherlands

e-mail: T.Gritsun@utwente.nl

\section{Introduction}

Dissociated neuronal cultures provide a useful platform to study behavior and development of biological neural networks (NNs). Deprived from external inputs neural cultures develop electrical activity patterns "on their own", showing several specific features. The most striking one is the phenomenon of more or less regular network bursts (NBs), i.e., almost simultaneous firing of many neurons in a relatively short time window. These spontaneous NBs have been subject of many experimental studies; see for example (Kamioka et al. 1996; Jimbo et al. 2000; Van Pelt et al. 2004b; Stegenga et al. 2008). Statistics of recorded bursting events are usually provided by averaging of the intra-burst firing rate profiles over long-term recordings. Usually, NB firing rate profiles have a sharp rising slope, a single maximum and a relatively slower descent. During cultured network development burst profiles undergo several changes. In particular, during the second week in vitro they become wider and higher in firing rate. The third week is usually characterized by reduction and stabilization of NB firing rate and NB width (Van Pelt et al. 2004a; Wagenaar et al. 2006; Le Feber et al. 2007; Stegenga et al. 2008).

It is well known that artificial random recurrent neural networks (ARRNNs) with excitatory and inhibitory neuronal populations are able to exhibit synchronous activity (Amari 1972). Even though the introduction of biological complexity into ARRNN models does not enable rigorous proof of network synchronizability, widely used numerical simulations of plausible RRNN clearly expose this feature (see e.g., Hansel and Sompolinsky 1996; Tsodyks et al. 1998, 2000; Giugliano et al. 2004). The oscillatory nature of the network activity may originate from different properties presented in neural systems, e.g., Van Vreeswijk et al. (1994) studied the inhibitory nature of the network oscillation; 
Tsodyks et al. (2000) showed that the delayed activation of synaptic depression is responsible for the network oscillatory behavior; Giugliano et al. (2004) studied spike adaptation features.

The current state of neuroscience provides a wide range of realistic neuron and synapse models that may serve as building blocks for network models. In general, network models contain three sets of parameters: neuronal parameters (such as rheobase, spike-frequency adaptation time constant, etc.), synaptic parameters (such as weights, post-synaptic potential time constant, etc.), and network connectivity parameters (number of connections per neuron, transmission delays, etc.). Most models proposed in literature describe network wide neuronal activity. These models are usually focusing on accuracy of their elementary units. For example, studies on network behavior have shown the influence of neuronal features (like spike-frequency adaptation; Van Vreeswijk and Hansel 2001; Giugliano et al. 2004) and synaptic features (such as synaptic decay times and depression parameters; Tsodyks et al. 1998; Wang 1999; Tsodyks et al. 2000; Fuhrmann et al. 2002; Brunel and Wang 2003; Wiedemann and Luthi 2003) on population rhythms. Many researchers supplement their models with additional equations and parameters for better mimicking neurons and synapses while trying to copy collective network behavior. However, often the importance of more global network parameters, such as number of connections per neuron and transmission delays (the time delay between pre-synaptic spike and post-synapse response) are disregarded. Many studies are restricted to sparse networks, up to a hundred connections per neuron; most of them use short or zero transmission delays (Mehring et al. 2003; Persi et al. 2004; French and Gruenstein 2006). However, integrated synaptic input from the network affects the network activity regime (see for example Brunel and Hakim 1999; Brunel and Wang 2003). This integrated input directly depends on the number of synaptic connections, transmission time delays of incoming spikes, and the excitatory/inhibitory ratio. While the effects of the first two parameter sets (neuronal and synaptic features) have been studied extensively, the influence of network parameters has been investigated less explicitly and is usually covered under the mask of Gauss-distributed noisy currents that emulate a realistic input from a large network (see for example Giugliano et al. 2004). However, taking into account that synchronous network bursts occur frequently, we may expect a big influence of network parameters on NB profiles.

The ranges of many network parameters are known. Neurons in cortical networks receive several thousands of synaptic inputs in vivo and about one thousand in vitro (Van Huizen et al. 1985; Ichikawa et al. 1993), delays may exceed the 40ms range in vivo (Swadlow and Waxman 1975) or 20-ms in vitro (Muller et al. 1997). Moreover, several experimental studies on synaptogenesis in cortical neuronal cultures show that network activity is correlated with the average number of synapses per neuron (further referred to as connectivity; Habets et al. 1987; Muramoto et al. 1993). Because connectivity changes during maturation of cultures, we cannot solely rely on neuronal and synaptic adaptation dynamics to describe the shapes of NB profiles over the life time of cultures.

The second issue we address in this study is emergence/origin of spontaneous activity. The literature provides two main sources, namely 'synaptic noise' (see for example Hubbard et al. 1967) and rhythmic pacemaker cells. The first one is most widely used in modeling studies and is well characterized by random (Poisson-distributed) synaptic inputs (Destexhe et al. 2004). The less used 'pacemaker-driven' approach is based on intrinsically active cells, as described by Latham et al. (2000a,b). Recent modeling studies showed that theoretically both the approaches can provide robustness of network bursting behavior in noisedriven (Nesse et al. 2008) and pacemaker-driven networks (Vladimirski et al. 2008). However, Vladimirski et al. (2008) showed that homogeneous noise-driven networks produced fragile rhythmic bursts. It is an open question which of these mechanisms causes spontaneous activity in neuronal cultures. To answer this, we simulated both 'noise-driven' and 'pacemaker-driven' neuronal networks, and we compared the results with experimental data.

The neuron and synapse models that we used may both include adaptation features (Izhikevich 2003; Markram et al. 1998). In this article, we will verify whether certain combinations of network parameters result in realistic burst profile parameters (as acquired from neuronal cultures), using either activity-independent or activity-dependent synapses with heterogeneous distribution of synaptic efficacies. Furthermore, we will investigate the sensitivity of burst profiles to network parameters.

\section{Methods}

\subsection{Simulation model}

Most modelers use conductance or circuit-based neuron models, like the Hodgkin-Huxley or integrate-and-fire neurons, respectively, because their parameters are biophysically meaningful. A drawback of these models is their high computational load due to the short simulation step needed (in the order of $0.1 \mathrm{~ms}$ ). Since this study focuses on network parameters, we chose to use the computationally more efficient canonical neuron model by Izhikevich (2003), which is able to reproduce the whole range of anatomical variability of basic cortical neurons as described by Toledo-Rodriguez et al. (2003). This model has four independent dimensionless parameters that determine the spiking behavior of the neuron. To set the values of these parameters we used the same 
normal distributions in all simulations. Thus, we were able to compare and interpret simulations resulting from changing network parameters only. Membrane potentials for all neurons were calculated using equations provided in Appendix A. We regarded the Izhikevich neuron model as a black box that has been demonstrated to provide realistic neuronal firing patterns.

As for synaptic kinetics, one can choose to use either an activity-independent model in favor of simplicity, or a biologically more realistic activity-dependent model. We aimed to compare the results of both the approaches. Again, we used well-known, existing models to describe synaptic behavior. For simplicity and unambiguous interpretation of the simulation results, we first used activity-independent synapses, therein comparing 'noise-driven' versus 'pacemaker-driven' network.

We compared two methods to ignite activity in the network models: noise injection or intrinsic 'pacemakers'. In case of noise-driven networks, the (random) noise injection may mimic synaptic or membrane noise. We adopted the method from Destexhe et al. (2004) which was validated in experimental studies on cortical slices. Each neuron received a Poissonian spike train $(J)$ of rectangular 1-ms pulses, whose amplitude was normally distributed between 0 and $8 \mathrm{mV}$. In response, neurons showed fluctuations in their membrane potential $(v)$ which yielded normally distributed values between -78 and $-55 \mathrm{mV}$. These values agree with experimental distributions (see Fig. 3C in Destexhe et al. 2004). We assume that noise frequency can change drastically during cell development; therefore, the mean rate of synaptic noise $J$ was varied in a wide range between 0 and $1 \mathrm{kHz}$ per neuron.

The second method ('pacemakers') is supported by several experimental studies, namely the presence of: (i) endogenously active cells as described by Latham et al. (2000a), (ii) 'privileged' neurons in neural cultures (Eytan and Marom 2006, or (iii) spontaneous burst initiation zones (Feinerman et al., 2007). In this part noise was set $J=0$ for all $i$ (see Eq. 1). Without input from other neurons the pacemaker neurons fired regular spikes with frequencies ranging from 0 to $0.26 \mathrm{~Hz}$.

Next, when the most suited mechanism for spontaneous activation was determined, we replaced the activity-independent synaptic model by an activity-dependent one. We used the adaptive (frequency dependent) synapse model by Markram et al. (1998), which mimics short-term facilitation and depression between heterogeneous [excitatory $(E)-$ inhibitory $(I)$ or $I-E]$ and homogeneous $(E-E$ or $I-I)$ synapses, respectively (see Appendix B for details). Thus, in our first simulations the synaptic weight range was constant, and in later simulations it was allowed to vary according to a well-established short-term plasticity (STP) model (Markram et al. 1998; Markram 2000). To mimic adaptive interaction between cortical neurons we used their phenomenological model and parameters, which are suggested to be responsible for setting population rhythms (Tsodyks et al. 1998, 2000). Thus, we again applied an existing model as a black box, to fully focus on the effects of network parameters on network-wide firing characteristics. The following sections describe the simulation design in more detail.

\subsubsection{Network parameters}

We used a random scale-free network model with recurrent connections. The NN model generates the following system of equations in general form:

$x_{i}(t+\mathrm{d} t)=\left\{\begin{array}{ll}1, & \text { if } f_{i}\left(v_{i}, w_{i j}, x_{j}\right) \geq 30 \mathrm{mV} \\ 0, & \text { otherwise }\end{array}\right.$,

where

$f_{i}\left(v_{i}, w_{i j}, x_{j}\right)=v_{i}(t)+J_{i}+\sum_{j=1}^{n} w_{i j} \cdot e_{i j} \cdot x_{j}\left(t-l_{i j}\right)$

Here $x_{i}$ is the generated firing pattern in a receiving neuron $i, x_{j}\left(t-l_{i j}\right)$ is the state of a transmitting neuron $j(i, j=$ $1,2, \ldots, n$, where $n$ is the total number of neurons). $J_{i}$ corresponds to synaptic noise, independent for different $i$ 's; the $v_{i}(t)$ term represents the nonlinear dynamics of the neuronal membrane potential of neuron $i$ and $\mathrm{d} t$ is the simulation step. The summation on the right-hand side of (1) represents input from the network and incorporates square, sparse $\mathbf{W}$ and $\mathbf{L}$ matrices and a matrix $\mathbf{E}$, whose elements are defined as follows. $e_{i j}$ is -1 if $j$ corresponds to an inhibitory neuron and 1 otherwise; $l_{i j}$ is the latency between a spike on neuron $j$ and the post-synaptic potential on neuron $i$; the synaptic weights are denoted by $w_{i j}$. First, activity-independent values for $w_{i j}$ were used, which were later replaced by activity-dependent values as described in Appendix B.

Now we assign values to $w_{i j}, l_{i j}$, and $e_{i j}$. The actual number of synaptic couplings from neuron $j$ is the number of nonzero elements per column in $\mathbf{W}$. Connectivity $K_{\max }$ was defined as the maximum number of couplings per neuron. All neurons had a number of synapses, taken from a normal distribution $\left(0, K_{\max }\right)$. To the nonzero elements $l_{i j}$ in latency matrix $\mathbf{L}$, we assigned values in the range $0-D_{\max }$ (maximal transmission delay), taken again from a normal distribution $\left(0, D_{\max }\right)$. Excitatory fraction $R$ is the percentage of excitatory neurons in the network.

In batch simulations we subsequently varied the parameters $K_{\max }, D_{\max }$, and $R$ while keeping the other parameters constant.

Only broad ranges for these network parameters can be found in literature. For instance, $K_{\max }$ may have values up to several thousands of synaptic contacts. In order to set realistic connectivity ranges we used approximations based on 
the mean number of synapses and neuron seeding density per volume unit, as observed in our own experiments or by others (Van Huizen et al. 1985; Habets et al. 1987). According to the experimental protocol in Habets et al. (1987), the neuronal seeding density was about 2000 cells $/ \mathrm{mm}^{2}$, which is similar to our experiments, and number of synapses per

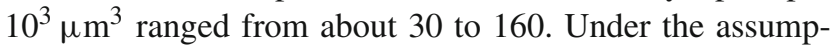
tion that a culture forms synapses in a monolayer of about $10 \mu \mathrm{m}$ (thickness of an average cell), the number of synapses per neuron can be up to 800. Ichikawa et al. (1993) showed that this number can be as high as 1100 (synapses per neuron). We used a connectivity range $\left(K_{\max }\right)$ up to 1100 . We estimated the transmission delays using normal distributions, which ranged up to $D_{\max }=25 \mathrm{~ms}$ according to experimental findings by Muller et al. (1997).

The peak values of the post-synaptic potential, referred to as synaptic weights $\left(w_{i j}\right)$, are usually distributed between 0 and $1 \mathrm{mV}$ (Magee and Cook 2000), but may reach values up to $12 \mathrm{mV}$ (Gibson 2003). According to these experimental data, we defined an inhomogeneous distribution for synaptic weights, which was composed of two sets. The first set represented the weights for the regular (nonpacemaker) neurons in both 'noise-' and 'pacemaker-driven' simulations and was normally distributed in the $[0,1] \mathrm{mV}$ range. In simulations with 'pacemakers,' we used two other sets of weights for corresponding neurons, either $[0,3] \mathrm{mV}$ or $[0,12] \mathrm{mV}$ intervals (normal distributions), in NNs with activity-independent or activity-dependent synapses, respectively. We varied the 'pacemaker' fraction between 4 and $16 \%$. We set the excitatory fraction $(R)$ in the range between 70 and $90 \%$, which is in agreement with many experimental studies (see for example Toledo-Rodriguez et al. 2003).

\subsection{Sensitivity and statistical analysis}

We performed batch simulations for gradual changes of the network parameters, as follows:

- Ratio of excitatory/inhibitory neurons or neural fraction $(R)$ was set to 70,80 , or $90 \%$.

- Connectivity ranged up to $K_{\max }$, which was set between 100 and 1100 in steps of 100 (i.e., neurons had random number of connections, normally distributed between 0 and $K_{\max }$.)

- Transmission delays ranged between 1 and $D_{\max }$ ms with a normal distribution, where $D_{\max }$ had values from 5 to 25 $\mathrm{ms}$, with a 5-ms simulation step.

- The total number of neurons was constant at $n=5000$.

\subsubsection{Output parameters}

As the experimental recordings were made using 60-electrode arrays we adopted this number to analyze simulated data. From every 1 min of simulation, subsets of 60 neurons $(R \%$ excitatory and $(100-R) \%$ inhibitory) were selected and NBs were detected whenever an average of 2 spikes per neuron appeared in a 10-ms time bin. We used a Gaussian filter (5-ms width) to smooth NB profiles.

Our network model produced bursts, which were characterized similar to the experimentally measured ones, as described by Stegenga et al. (2008). Three intra-burst parameters, namely maximum firing rate $(\mathrm{mFr})$, half-width of the rising slope (Rs), and half-width of the falling slope (Fs) were calculated as follows (see Fig. 1): $\mathrm{mFr}$ is taken at the burst peak, Rs and Fs are the intervals (in ms) between time at burst peak and time at $50 \%$ of burst height, of rising, and falling slopes of the main phase, respectively. The main phase is defined as the phase around $\mathrm{mFr}$. It is separated from a preand tail-phase by two local minima, each far below the $50 \%$ (of $\mathrm{mFr}$ ) level.

We aligned the main phases acquired from every simulation by their peak. Then we calculated the firing rate probability with $10-\mathrm{Hz}$ bin at every time step (1 ms) in a time window between $300 \mathrm{~ms}$ before and $300 \mathrm{~ms}$ after the peak. Finally, we calculated 7.5th, 92.5th percentiles (further referred to as lower and upper percentiles) and medians for every time step. And we followed the same procedure for NBs in every culture, where firing rate probability distributions occupied a very wide area because of a few outliers. We chose these percentiles as a fair compromise between a high percentage and a small area. In the Fig. 3A, we plotted the percentiles and medians against time (e.g., see black solid and dashed curves).

We studied NB profiles, which were calculated as a (smoothed) estimation of the array-wide firing frequency (Van Pelt et al. 2004b; Stegenga et al. 2008), without attempting to analyze neuron spiking behavior or explicit comparison with individual neurons in culture.

Network simulations were performed using $\mathrm{C}$ programs (MEX-files) in a Matlab environment (the MathWorks, Inc) on a PC compatible platform. We used Euler's method to integrate neuronal and synaptic model equations with $\mathrm{d} t=1 \mathrm{~ms}$ simulation step.

\subsection{Measured data}

Experimental data were obtained from six cortical cultures. Culturing, recording, and other experimental techniques were as follows. Cortical neurons were obtained from either newborn or E18 Wistar rats by trituration and chemical dissociation using trypsin. The cells were plated at a concentration of $10^{6}$ cells $/ \mathrm{ml}$, and allowed to adhere for $2 \mathrm{~h}$. Multi electrode arrays (MEAs) were coated in advance with polyethylene-imine to increase adhesion. The nonadhering cells were then removed by refreshing the medium, and $600 \mu \mathrm{l}$ of R12 medium was added. The resulting monolayer had a 
Fig. 1 a Example of the activity recorded from one of the cultures, represented in a spike raster plot. Each dot represents a recorded action potential at the indicated electrode. b Enlarged view of one of the bursts in (a). c Examples of network burst (NB) profiles acquired from one of the cultures (green and blue lines) aligned at the peaks of the main phases. Waves before and after the main phases are referred to as pre-phase and tail-phase, respectively (occasionally we also saw a flanking phase).

Characterization of the main phase by $\mathrm{mFr}$, Rs, and Fs are shown in one example (thick blue line). $\mathrm{mFr}$ is taken at burst peak, Rs and Fs are the intervals between burst peak and $50 \%$ of burst height, of the left and right sides of the main wave, respectively
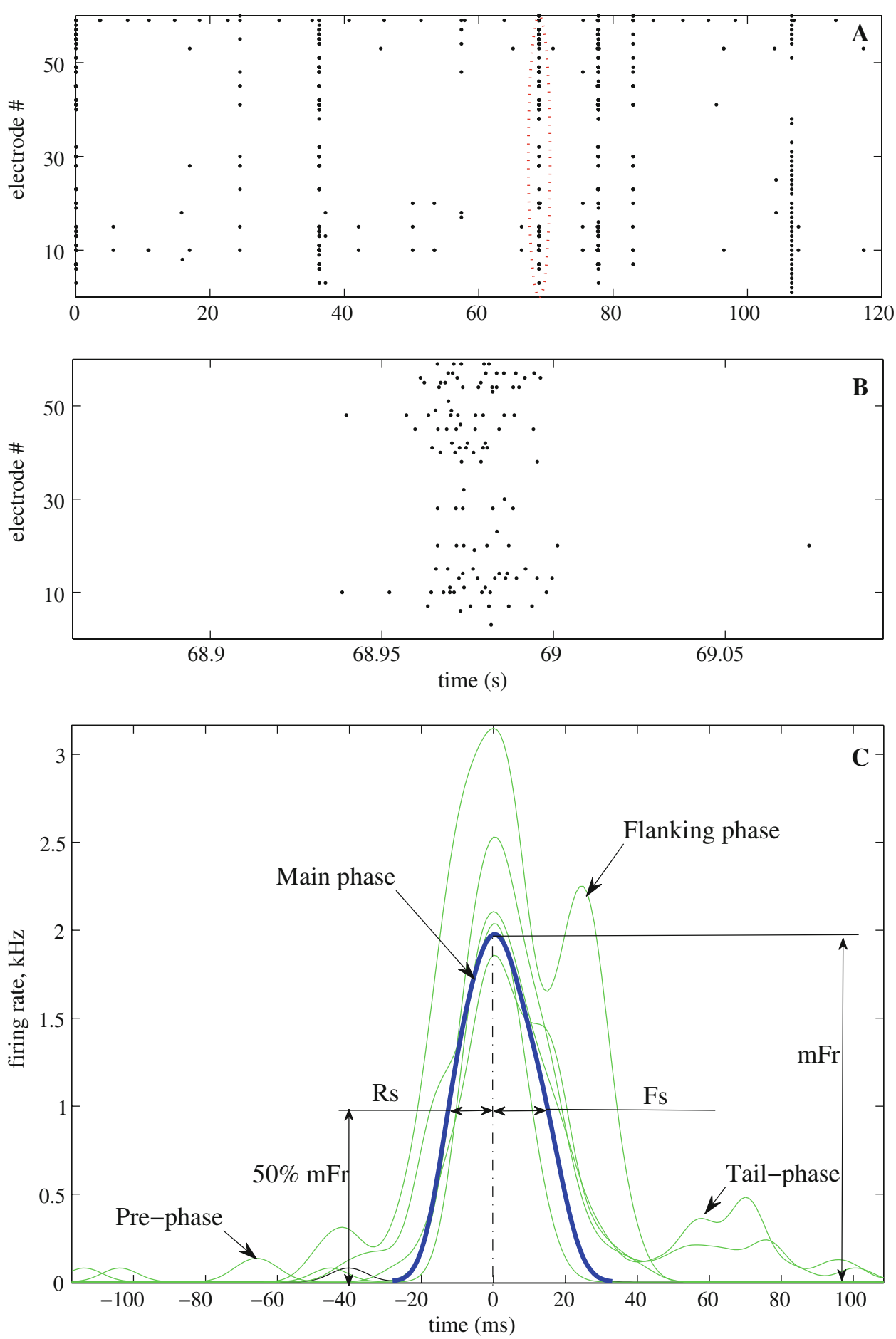

density of $\approx 5000$ cells $/ \mathrm{mm}^{2}$. Two-thirds of the medium was changed twice a week. The cultures were stored in an incubator at $37^{\circ} \mathrm{C}$, at a $\mathrm{CO}_{2}$ concentration of $5 \%$ and near $100 \%$ humidity.

We used a MC1060BC setup and MEAs from MultiChannel Systems GmbH. The MEA's had 60 Titanium-Nitride electrodes in an $8 \times 8$ square grid. The inter-electrode distance was $100 \mu \mathrm{m}$, and the diameter of the electrodes was $10 \mu \mathrm{m}$. The temperature was controlled at $36^{\circ} \mathrm{C}$ and $\mathrm{CO}_{2}$ concentration of $5 \%$ was maintained. Custom made LabView (National Instruments, Austin, Tx) programs were made to control data-acquisition (Stegenga et al. 2008). 
Table 1 Overview of the experimental data ranges

\begin{tabular}{|c|c|c|c|c|c|c|c|}
\hline \#Culture & 1 & 2 & 3 & 4 & 5 & 6 & Average \\
\hline Recorded DIVs & $14-18$ & $11-18$ & $12-17$ & $9-36$ & $10,15,16$ & 11,12 & \\
\hline Number of NBs & 1193 & 873 & 698 & 2482 & 547 & 534 & \\
\hline \multicolumn{8}{|l|}{$\mathrm{mFr}$} \\
\hline Mean (kHz) & 6.42 & 2.12 & 5.73 & 3.13 & 2.71 & 3.75 & 3.98 \\
\hline SD & 0.63 & 0.58 & 0.67 & 0.82 & 0.51 & 0.57 & 1.82 \\
\hline \multicolumn{8}{|l|}{ Rs } \\
\hline Mean (ms) & 13.23 & 7.48 & 15.85 & 15.37 & 11.39 & 11.42 & 12.5 \\
\hline $\mathrm{SD}$ & 5.99 & 0.87 & 5.67 & 5.6 & 4.5 & 4.8 & 4.8 \\
\hline \multicolumn{8}{|l|}{ Fs } \\
\hline Mean (ms) & 17.58 & 8.52 & 17.65 & 16.1 & 16.49 & 30.75 & 15.4 \\
\hline SD & 6.0 & 0.9 & 5.4 & 4.6 & 5.7 & 7.7 & 5.9 \\
\hline
\end{tabular}

$m F r$ maximal firing rate, $R s$ rising slope, $F$ s falling slope, $N B$ network burst, $D I V$ days in vitro, $S D$ standard deviation

\section{Results}

\subsection{Statistical analysis of the experimental data}

We were primarily interested in studying and comparing collective behavior in experimental and simulated NNs by their NB characteristics. This included analysis of three profile features (mFr, Rs, and Fs) and how they depended on the network parameters, connectivity, excitatory fraction, and transmission delays, parameters that develop in biological NNs during culture growth. Figure 1 shows a typical example of the spike raster plot (A and B) and several examples of NB profiles (C) acquired from one of the recorded cultures, aligned at the maximum of the main phase. In general, we divided whole NB profiles into a main phase, which in most cases lasted around $80 \mathrm{~ms}$, and additional phases before and after the main phase, referred to as pre-phase and tail-phase, respectively. In spite of rich profile variation, the upper part (above $50 \%$ of maximum of the main phase) appeared to be statistically stable and, thus, appropriate for quantitative characterization of synchronous network-wide activation. The bell-shaped upper part was characterized by three parameters: $\mathrm{mFr}$, Rs, and Fs. Most of the experimental NB profiles were symmetric (with equal Rs and Fs) or leftshifted (with slightly longer Fs than Rs). Only a few percent of the profiles had longer Rs than Fs.

First, we pooled data from all cultures and determined first-order statistics, i.e., average values and data ranges. In general, our experimental data showed the following ranges: $\mathrm{mFr}$ ranges from 0.8 to $8 \mathrm{kHz}$, Rs ranges from 6 to $40 \mathrm{~ms}$, and Fs ranges from 7 to $47 \mathrm{~ms}$. Mean and SD values are shown in Table 1. We used these data for model validation.

Figure $2 \mathrm{a}-\mathrm{c}$ shows the distributions of $\mathrm{mFr}$, Rs, and Fs, respectively, for each culture. Distributions have different shapes and variances, with overlapping ranges. For exam-
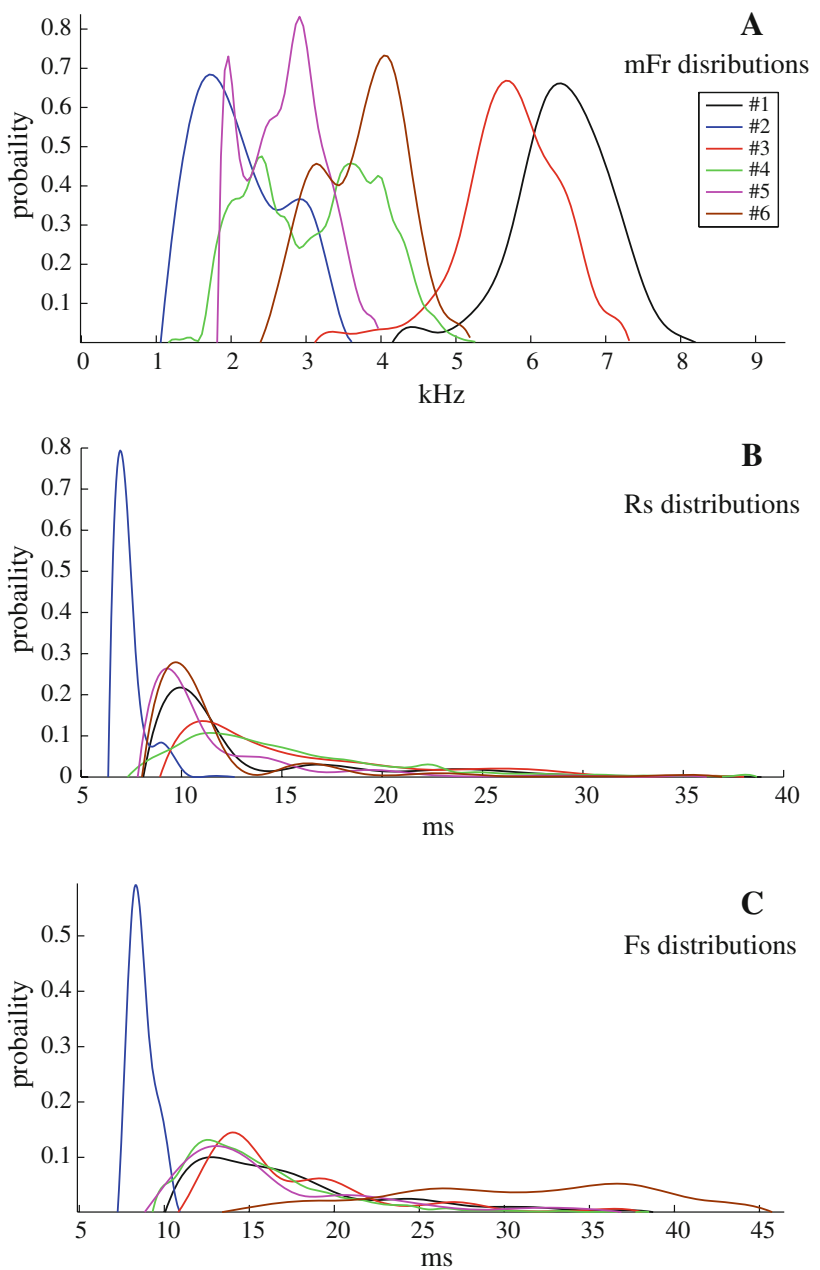

Fig. 2 Overview of experimental parameters. Distributions of $\mathrm{mFr}(\mathbf{a})$, Rs (b), and Fs (c) acquired from six cultures marked by colors. Note that Fs distribution curves are shifted to the right with respect to corresponding Rs curves 

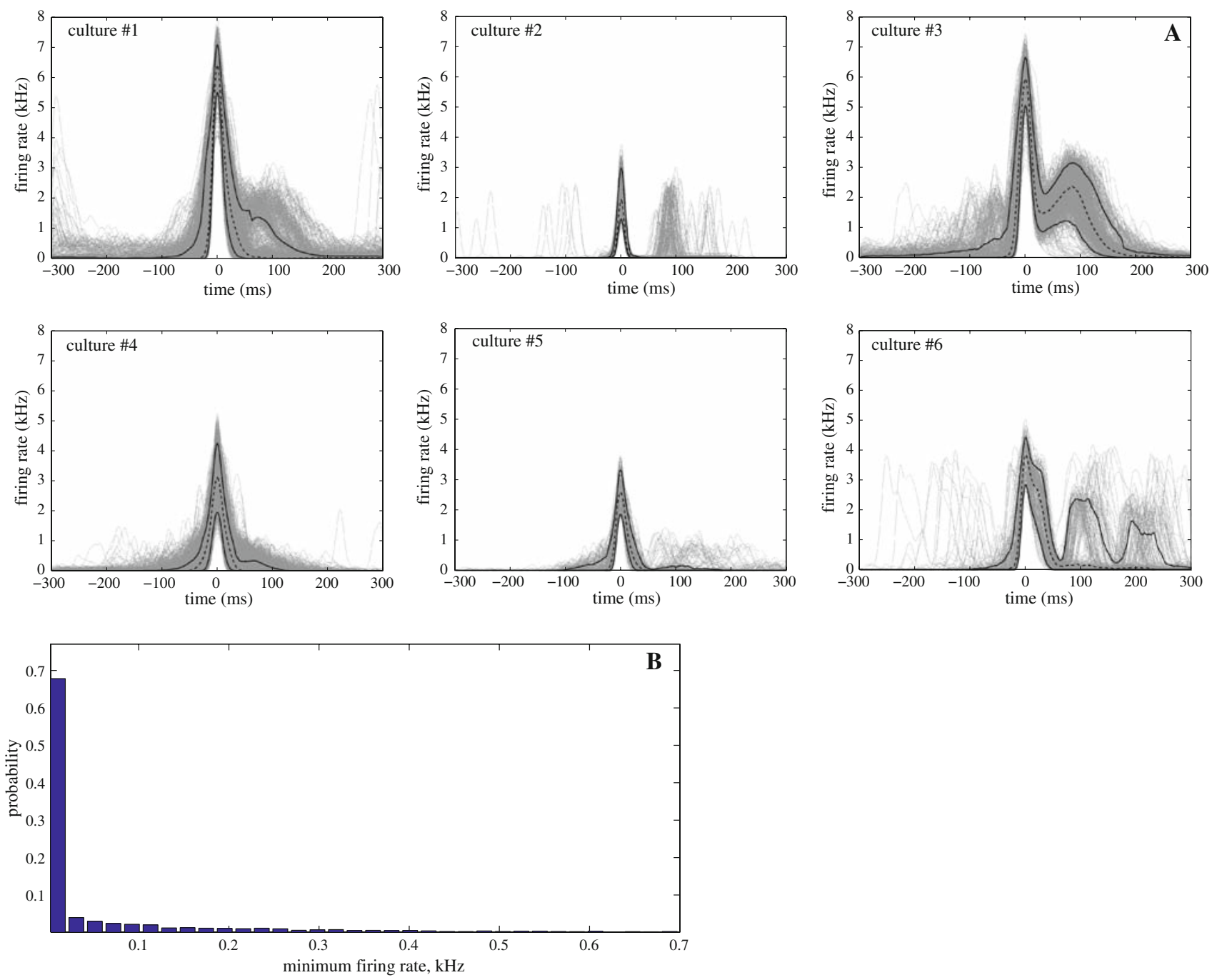

Fig. 3 a Overview of experimental NB profiles from six recorded cultures. For each culture we composed profile sets from NBs aligned by their peak (gray lines) and calculated 7.5 th and 92.5 th percentiles (black solid lines) and medians (dashed lines). b Distribution of the

ple, $\mathrm{mFr}$ distributions acquired from cultures \#1 and 3 have similar (normal-like) shapes with SD around $0.66 \mathrm{kHz}$, but different mean values. Rs and Fs distributions also show several similar gamma-like shapes (see Fig. 2b).

In a search for the origin of the spontaneous NB, we examined the starting point of the rising slope of the experimental main phase. Profiles recorded from each culture were pooled into separate sets, which were used to calculate median and percentile curves. Figure 3 shows six sets of experimental curves. We chose to use median values instead of average curves because of the asymmetric nature of the firing rate distributions. For all recorded cultures, we found that the median dropped to zero in the time interval $[-50:-15]$ $\mathrm{ms}$ before the peak. This means that in this interval there is no minimum level of activity (a kind of rheobase at network minimum firing rate in $[-50:-15] \mathrm{ms}$ time interval before the peak of the global profile. The histogram was calculated over all detected NBs $(N=6327)$. Most of the NBs show the firing rate minimum in the first $(20 \mathrm{~Hz})$ bin

level) required to initiate a NB. Figure $3 b$ shows the distribution of minimum firing rate at $[-50:-15] \mathrm{ms}$ time interval before $\mathrm{mFr}$ acquired from all recorded profiles. This indicates that our neuronal cultures did not require maintained network activity for main phase initiation. To compare measured and simulated data, we conducted similar analysis, in the same time interval, on simulated NB profiles.

In the scope of this article, we investigated three network models. The network model, responding to gradually increasing internal noise (1), 'pacemaker-driven' network activity, with either activity-independent (2) or activity-dependent (3) synapses. In subsequent sections, we will study which model best mimics the origin of the NB main phase and we will analyze the sensitivity of NB profile features to the network parameters. 
Fig. 4 Example of firing patterns in the 'noise-driven' neural network. Top simulated spiking train segments that correspond to the network activity induced by noise injections indicated on the $x$-axis of the bottom plot (black arrow indicates threshold of changing dynamics beyond which NBs were detected; the segments show $400 \mathrm{~ms}$ of data, taken from much longer simulations). Bottom the firing rate curve (mean: dashed line; standard deviation: bold line, calculated with 100 -ms bins) as a function of injected noise frequency per neuron. The mean firing rate for a set of 60 neurons was calculated from all spikes emitted during simulation steps with a constant frequency of injected noise

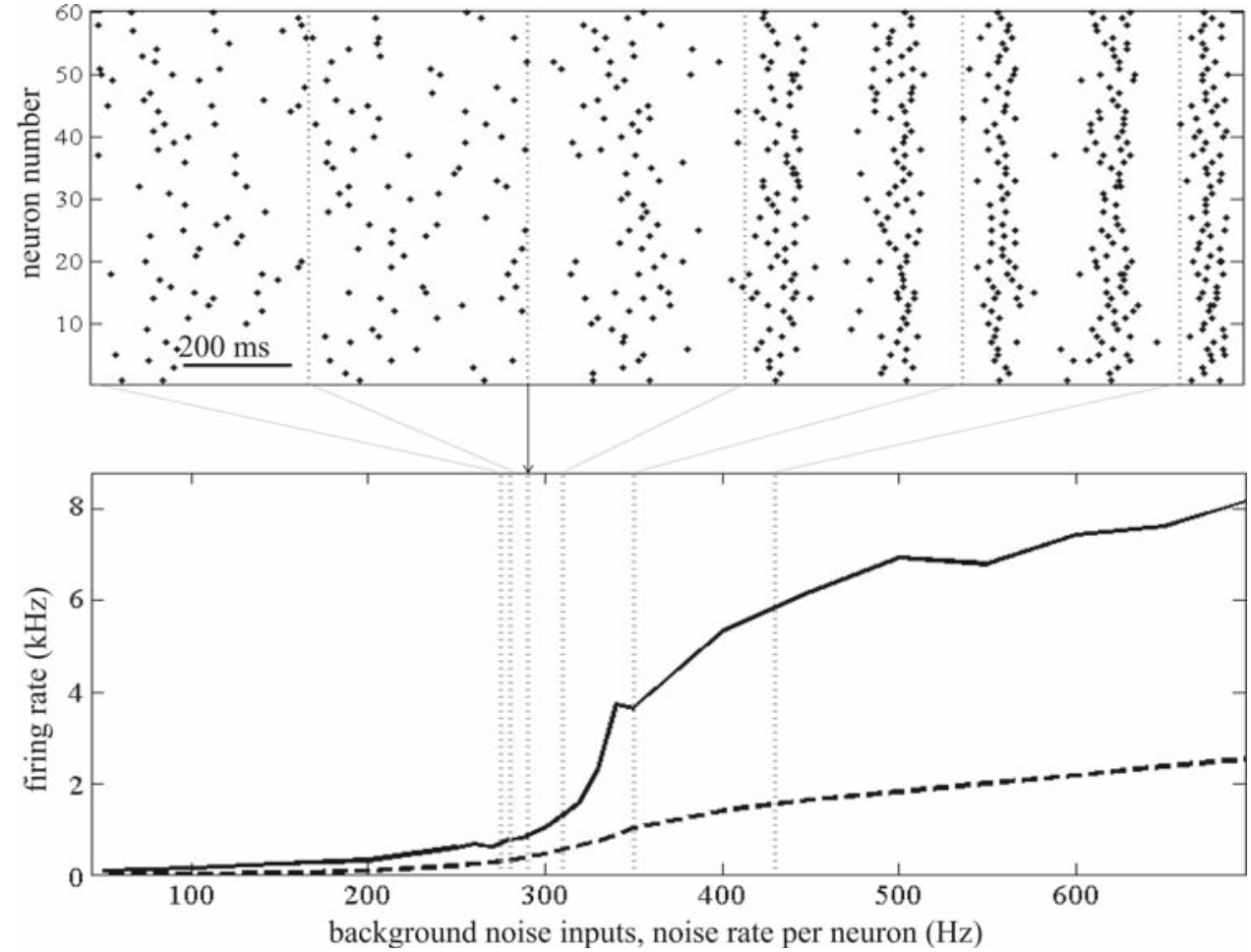

3.2 Network model response to gradually increasing external noise

First, we tested the model response to injected Poisonian spike trains with gradually increasing mean frequency, while keeping all other parameters constant. Relatively low-rate noise evoked asynchronous random firing (Fig. 4, top plot, see first two segments), whenever the amplitude of the injected stimulus exceeded the neuronal rheobase of the receiving neuron. Then, beyond a certain noise rate (see Fig. 4, arrow), network models switched to synchronized bursting behavior (Fig 4, see segments on the right of the arrow).

We characterized this change in the collective behavior by changes in mean firing rate of the NB (calculated over time periods with constant noise-stimulation rate) and its fluctuations (SD). Firing rate curves are depicted in Fig. 4 (bottom plot). We observed a frequency threshold where the network behavior changes, i.e., NBs appeared. Bursts originated while ongoing background noise recruited new neurons. In spite of the high amplitude of the noise spikes (up to $8 \mathrm{mV}$ ), Fig. 4 shows that networks required a noise rate higher than 290 $\mathrm{Hz}$ /neuron to produce NBs. Then, the network firing rate rose gradually and NB profiles developed in an exponential manner. To ensure burst-dominated network activity, we set the rate of injected noise to $330 \mathrm{~Hz}$ and ran batch simulations with all possible combinations of the parameters $K_{\max }$, $D_{\max }$, and $R$. Simulations with $R \geq 80 \%$ and $K_{\max } \geq 600$ produced NBs with an mFr range broader than experimental. Then, we further analyzed simulations using combinations of $K_{\text {max }}, D_{\text {max }}$ and $R$ that yielded NBs whose mFr, Rs, and Fs values matched experimental ranges. In general, this was for simulations with $R=70 \%$ and $D_{\max }$ from 10 to $20 \mathrm{~ms}$.

The common characteristic of all NB profiles produced by 'noise-driven' NNs was the long pre-phase (from about $-150 \mathrm{~ms}$ before $\mathrm{mFr}$ ), gradually developing into the rising slope of the main phase. These simulations always produced NBs whose median curve had a long and elevated onset of the rising slope, while the rising slope in experimental medians had no long or elevated onset. Figure 5a shows about $800 \mathrm{NB}$ profiles and their median and percentile curves from a NN simulation with $K_{\max }=1000, D_{\max }=15 \mathrm{~ms}$ and $R=70 \%$. In these simulations, in general, the lower percentile curves rose before or around the same time period of $-100 \mathrm{~ms}$ before the mFr peak as the upper percentile curves of the experimental data. Of all cultures, culture \#4 showed the longest pre-phase and the widest percentile area on the rising slope. The inset in Fig. 5 allows to compare experimental and simulated percentile curves, acquired from culture \#4 and the 'noise-driven' simulation (the same as in the main plot), respectively. Similar to the experimental part, we calculated minimum network activity in the $[-50:-15]$ ms time interval before the NB peak. We found that 'noise-driven' NN required an elevated and maintained network firing rate at about $0.26 \pm 0.12 \mathrm{kHz}$ (mean $\pm \mathrm{SD}$ ) to initiate a main phase. The histogram in Fig. 5B represents the typical distribution 
Fig. 5 a A typical example of a NB profile set (gray lines) acquired from a 'noise-driven' $\mathrm{NN}$ simulation with

$K_{\max }=1000, D_{\max }=15 \mathrm{~ms}$, and $R=70 \%$, which resembled the $\mathrm{mFr}$ range of culture \#4. The black dashed line corresponds to the median and black solid lines are the percentiles. Inset shows the simulated (red lines) and experimental (black lines) percentiles and medians acquired from culture \#4. b Distribution of the minimum firing rate in the $[-50:-15] \mathrm{ms}$ time interval before the main peak of the NB profile. The histogram was calculated from all simulated NBs shown in (a). c Sensitivity curves of Rs (black solid line) and Fs (blue dashed line) in simulations $w i$ th changing connectivity $\left(K_{\max }\right)$. Data were collected from simulations with $\mathrm{mFr}$ in the experimental range $(R=70 \%$, $\left.D_{\max }=[10: 20] \mathrm{ms}\right)$
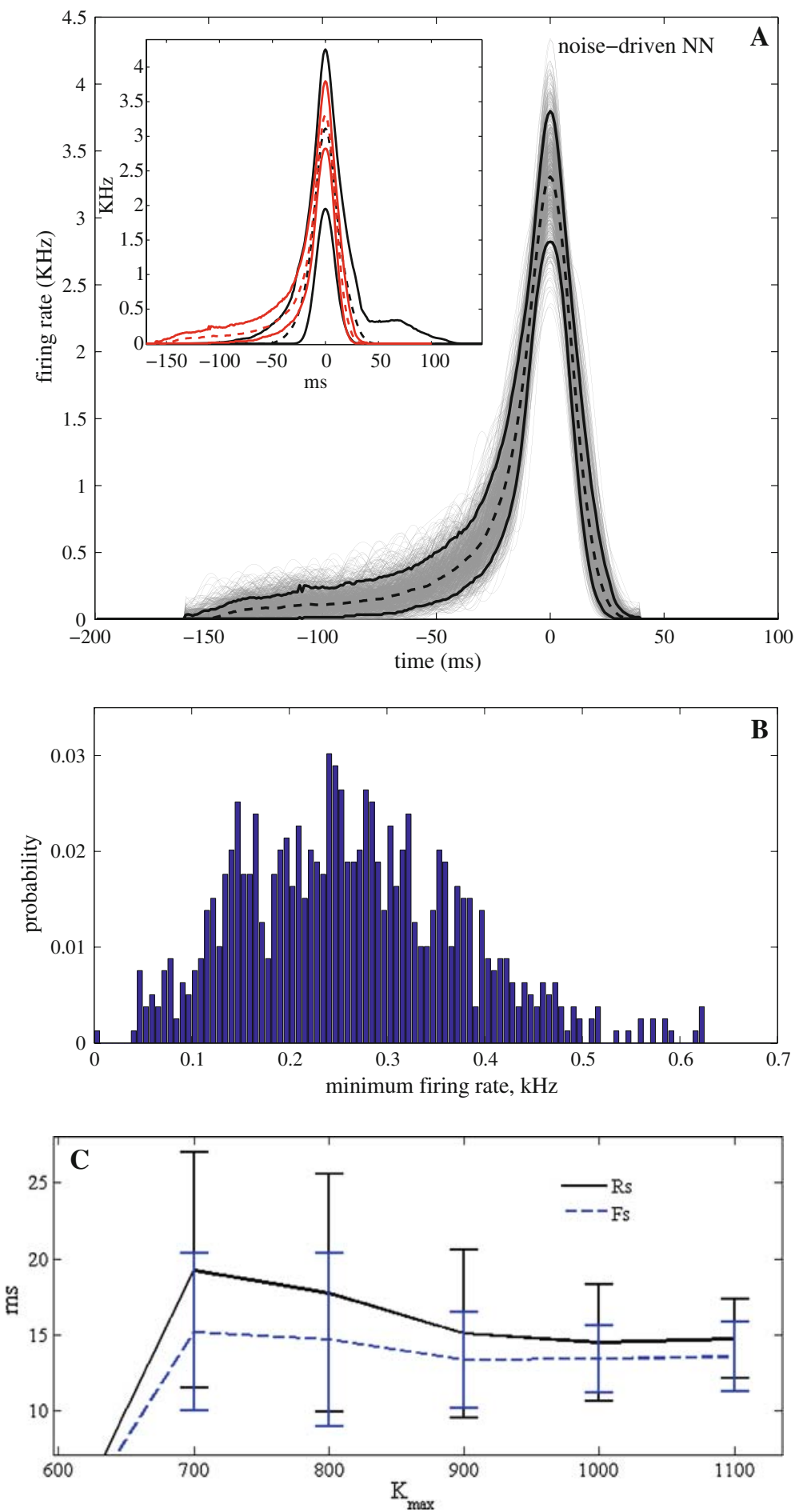

of minimum firing rates within that time interval. Furthermore, while developing into the main phase, this elevated onset increased Rs values, making it unnaturally longer than Fs. Figure 5c shows Rs and Fs sensitivity curves (note that Rs $>$ Fs, whereas experimentally Rs $<$ Fs).

\subsection{Networks with 'pacemaker-driven' activity}

In 'pacemaker-driven' simulations, we added intrinsically active neurons and ran these models without any noise injection. Pacemakers ignited network activity, as they were able 


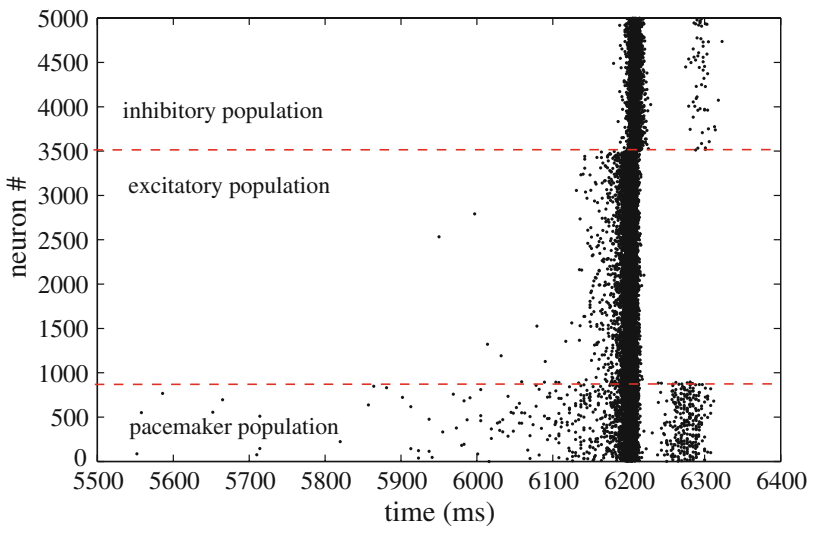

Fig. 6 A typical example of a spike raster plot produced by a 'pacemaker-driven' $\mathrm{NN}$ model with activity-independent synapses, $K_{\max }=$ $1100, D_{\max }=15 \mathrm{~ms}, R=70$ and $16 \%$ of pacemakers. Note that the pacemaker population fired first, which provided an input to the network strong enough to generate a NB

to fire with small or no input from other neurons in the network. In order to produce any activity, network models needed different amounts of 'pacemakers' (with ordinary [0, 1] $\mathrm{mV}$ synapses), ranging from 12 to $16 \%$ of the total number of neurons in the network, depending on the parameters $K_{\max }, D_{\max }$, and $R$. In the simulations, we observed that the firing pacemakers provided input to the network, and occasionally reached a level of synchronicity strong enough to generate NBs that show striking resemblance to NB profiles acquired from experimental recordings. There was reasonable likeliness to reach such level of synchronization because the pacemakers were defined as a homogeneous population, with comparable intrinsic firing frequencies. Figure 6 shows a typical spike raster plot acquired from one of these simulations.

Then we reduced the amount of pacemakers to about $4 \%$, using stronger synaptic connections $([0,3] \mathrm{mV})$ for the 'pacemakers' (other parameters were the same). Similar to the previous section, simulations whose $\mathrm{NB}$ profiles showed $\mathrm{mFr}$, Rs, and Fs values in the experimental ranges were selected for further analysis.

These NN models produced NB profiles with several realistic features: short pre-phase, symmetric and left-shifted main phases in most cases, and occasionally even rare features like a flanking phase on the slope of the main phase. As before, we focused on the rising slope of the median and percentile curves. We found that 'pacemaker-driven' simulations better reproduced experimental NB profiles than 'noisedriven' simulations. For 5 out of 6 experimental percentile curves we were able to find simulated percentiles with a rising slope that fitted into the experimental range, except for percentiles acquired from culture \#2, which had a very short start-up interval of the rising slope ( -20 to $-15 \mathrm{~ms}$ before the NB peak, see Fig. 3 box 2). Figure 7a shows a typical example of NB profiles along with their median and percentile curves produced by the NN with $K_{\max }=1100, D_{\max }=15 \mathrm{~ms}$, $R=70$ and $14 \%$ of pacemakers (all profiles). The corresponding median curves of both simulated and experimental data rose at about the same time (see inset in Fig. 7). 'Pacemaker-driven' NNs did not require elevated and maintained activity to initiate a NB. Here, distributions of minimum network activity in the $[-50:-15] \mathrm{ms}$ time interval before the NB peak showed better resemblance to experimental data than those from 'noise-driven' simulations. Figure $7 \mathrm{~b}$ shows a typical distribution of the minimum firing rate acquired from the simulated data shown in Fig. 7a.

The simulations described so far show that 'pacemakerdriven' NNs produce NB profiles that better resemble experimental profiles than 'noise-driven' simulations. Therefore, we chose this model for further simulations. Both 'noise-' and 'pacemaker-' driven models, with most combinations of realistic $K_{\max }, D_{\max }$ and $R$ values, produced profiles whose $\mathrm{mFr}$ values ran beyond the experimental range. Figure 8 shows several examples of sensitivity curves. We found that networks with short $D_{\max }=5 \mathrm{~ms}$, high $R$ (from 80 to 90\%) and $K_{\max }>800$ increased $\mathrm{mFr}$ drastically, with subsequent "explosions" of the network activity (dot-dashed and solid lines with $K_{\max }$ above 700 and 1000, respectively, in Fig. 8a). Rs and Fs values fell inside the experimental range (gray background in Fig. 8b). We found that mean values of Rs and Fs increased with bigger $D_{\max }$ or $K_{\max }$ (not shown here).

\subsection{Simulations with activity-dependent synapses}

Next, we introduced frequency-dependent synapses. Strengths of synapses leading from pacemaker neurons were set to [0:12] $\mathrm{mV}$ in order to ignite sufficient activity for sensitivity analysis and statistical comparison with experimental data. All other model parameters remained the same.

Compared to the simulations with activity-independent synapses the results clearly improved. Most of the network parameter combinations led to acceptable ranges for $\mathrm{mFr}$, Rs, and Fs in simulated NB profiles. Only the simulations with critical combinations of network parameters $\left(K_{\max } \geq 900, D_{\max } \leq 10 \mathrm{~ms}\right.$, and $R>80 \%$ ) produced $\mathrm{mFr}$ values which ran beyond the experimental range. For example, simulations with $D_{\max }=5$ and $10 \mathrm{~ms}, R=90 \%$, ran up to $\mathrm{mFr}=17 \mathrm{kHz}$, in densely connected NNs (with $K_{\max }=900$ and higher). Other combinations produced values that matched the specific ranges acquired from individual neuronal cultures. Table 2 shows the total mean and SD values (critical $K_{\max }, D_{\max }$ and $R$ combinations were excluded). In other respects these simulations showed similar results as previous ones. Figure 9a shows a typical example of the NB profiles produced by this network model (with $K_{\max }=1000, D_{\max }=15 \mathrm{~ms}$ and $R=80 \%$ ). The inset shows median and percentile curves from this simulation and the experimental profile we used before. 
Fig. 7 a An example of a NB profile set acquired from a 'pacemaker-driven' NN model with activity-independent synapses,

$K_{\max }=1100, D_{\max }=15 \mathrm{~ms}$, $R=70$ and $14 \%$ of pacemakers. The black dashed line corresponds to the median and the black solid lines are the percentiles. The inset shows simulated (blue lines) and experimental (black lines) percentiles and medians acquired from culture \#4 as in Fig. 5a. b Distribution of the minimum firing rate in the $[-50:-15]$ ms time interval before the main peak of the global profile. The histogram was calculated from all simulated NBs shown in (a)
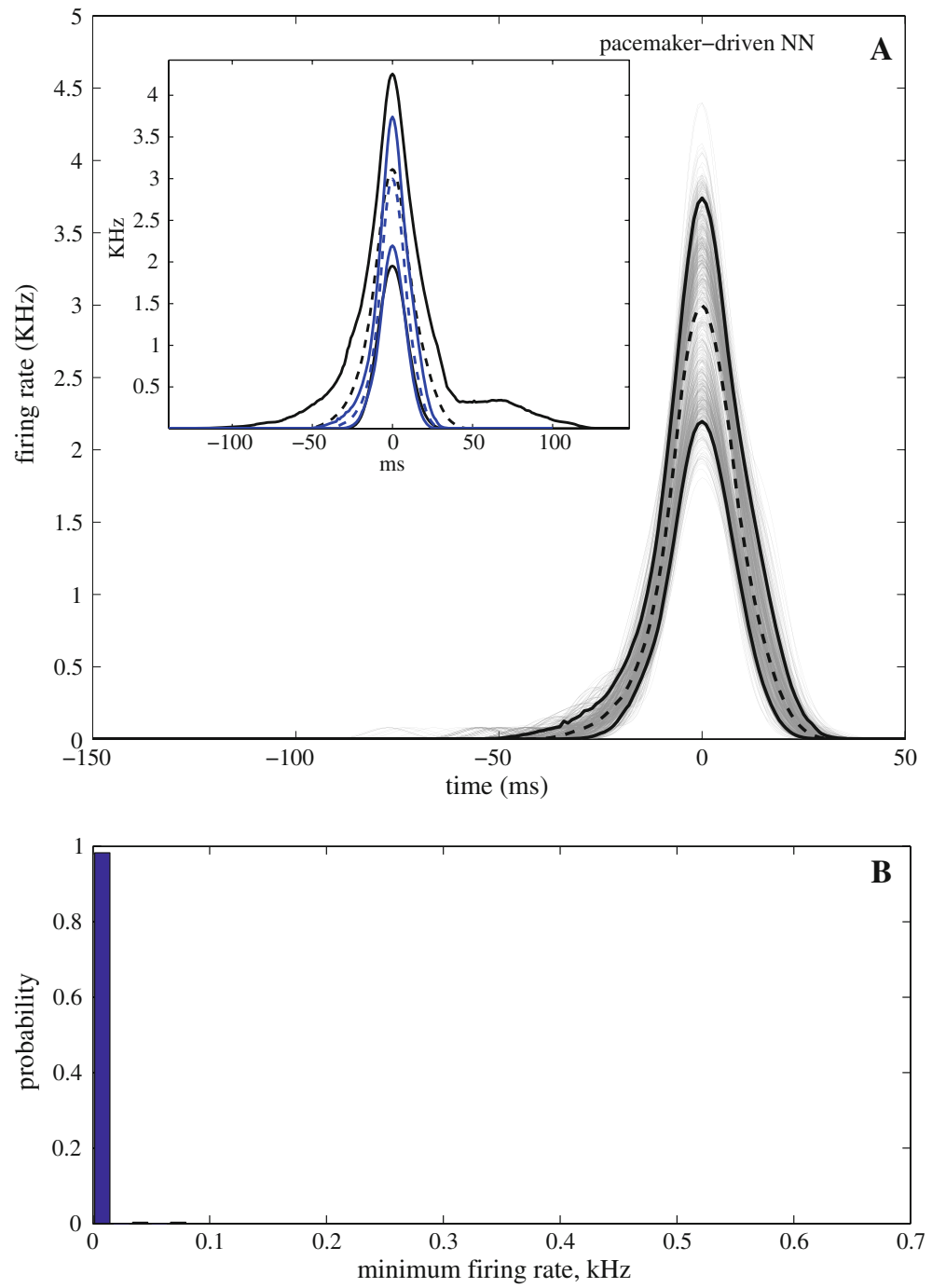

Table 2 Statistical data for all 'pacemaker-driven' NN simulations with activity-dependent synapses $\left(K_{\max }<900, D_{\max }>10 \mathrm{~ms}\right.$ and $R \leq$ $80 \%)$

\begin{tabular}{llcc}
\hline NB parameter & \multicolumn{2}{l}{ Average values } \\
\cline { 2 - 4 } & Range & Mean & SD \\
\hline $\mathrm{mFr}(\mathrm{kHz})$ & $0.8-8$ & 4.1 & 2.6 \\
Rs $(\mathrm{ms})$ & $7-33$ & 11.8 & 2.7 \\
Fs $(\mathrm{ms})$ & $8-39$ & 13.7 & 3.2 \\
\hline
\end{tabular}

Simulated Rs and Fs distributions were in the experimental range (Fig $2 b, c)$. Figure $9 b$ shows a typical example of Rs and Fs distributions collected from these simulations (note the gamma-like shapes).

Providing acceptable ranges of the main phase parameters and distribution shapes, these simulations allowed for analysis of sensitivity of $\mathrm{mFr}$, Rs, and Fs to the model network parameters. Figure 10 shows several sensitivity curves of NB profile features to the network parameters. In comparison to the $\mathrm{NN}$ models with activity-independent synapses, simulations with adaptive synapses yield a stable and smooth increase of $\mathrm{mFr}$ with increasing average network connectivity. Here, curves show a rising tendency, as in models with activity-independent synapses. However, now they do not show any explosion of activity. We also noted that $\mathrm{mFr}$ increased with higher $R$ (Fig. 10a, curves with different line-types) and smaller $D_{\max }$ (curves with different colors).

The Rs values were about $2-3$ ms smaller than Fs and changed similarly in response to changing network parameters. Figure 10b shows the sensitivity of Rs. In general, Rs and Fs showed a small increase with increasing $K_{\max }$ (e.g., highlighted dot-dashed magenta curve with SD bars), but in few cases they decreased with higher $K_{\max }$ (e.g., solid magenta curve). 
Fig. 8 Sensitivity curves of the NB profile parameters to connectivity $\left(K_{\max }\right)$ in 'pacemaker-driven' NN models with activity-independent synapses. Gray background areas correspond to the experimental mean $\pm \mathrm{SD}$. a Mean curves are plotted for $\mathrm{mFr}$. Note that $\mathrm{mFr}$ ran beyond the experimental range for certain combinations of the network parameters. b Rs and Fs mean curves (and SD bars) acquired from all simulations with $\mathrm{mFr}$ in the experimental range (again, gray area indicates experimental mean $\pm \mathrm{SD}$ )
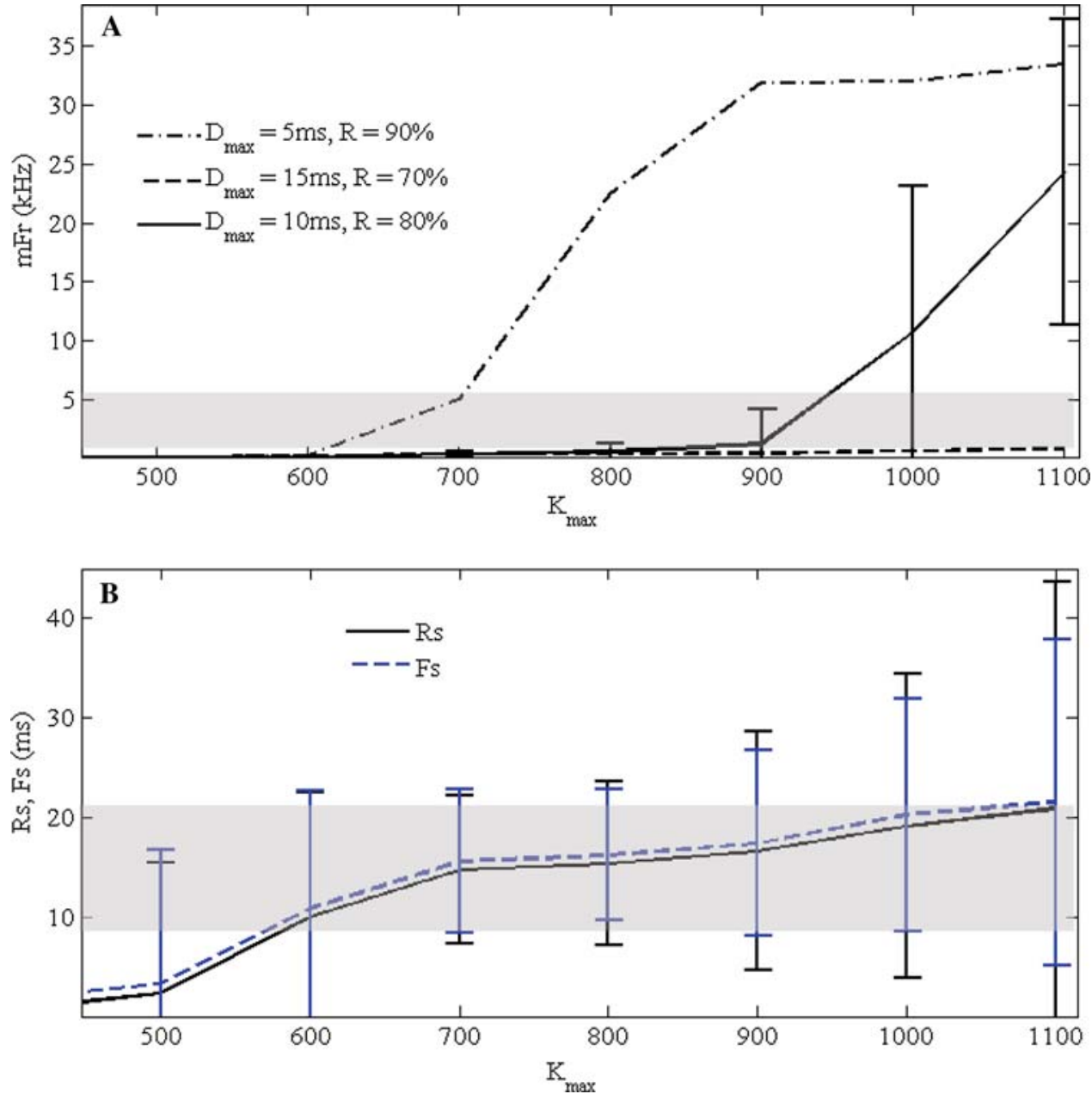

Changing $R$ has a larger effect on Rs and Fs. With $D_{\max }$ between 10 and $20 \mathrm{~ms}$, Rs and Fs increase with increasing $R$ (red, green, and magenta curves for $D_{\max }=[10,15,20] \mathrm{ms}$, respectively). With $D_{\max }=5 \mathrm{~ms}$, increased $R$ can result in a slight reduction of Rs and Fs (blue curves).

Increasing $D_{\max }$ causes negligible changes in Rs and Fs in simulations with low $R(70 \%)$, but becomes more noticeable with higher $R$. In general, increasing $D_{\max }$ causes an increase in Rs and Fs (dot-dashed curves).

\section{Discussion}

Spontaneous bursting behavior in cortical neuronal cultures has been a central issue in many recent experimental and modeling studies. In spite of the big repertoire of bursting patterns, there were several attempts to characterize NBs according to their profile features; see for example Van Pelt et al. (2004b); Wagenaar et al. (2006); Stegenga et al. (2008). Features become clear after statistical analysis of the experimental recordings; they may be explained via NN modeling and statistical comparison with experimental data. In this study, we mainly focused on the main phase of NB profiles, which represents the activation of most neurons in the network. Using analyses of both experimental and simulated data we addressed two main issues: onset of the main phase (1) and NB parameter sensitivity to changing network parameters (2). The first issue was studied by analysis of the rising slope Rs of the main phase. To study the second issue we quantitatively evaluated the experimental ranges of three NB parameters and analyzed the influence of the parameters $K_{\max }, D_{\max }$, and $R$ on simulated NB profiles.

We presented simulation results based on large networks with biologically realistic connectivity (up to 1100 connections per neuron), transmission delays (1-20 ms), and excitatory fraction (70-90\%), and analyzed their collective behavior in terms of synchronized NBs. We built our network models using existing neuronal and synapse models with experimentally estimated parameters. In this study, we focused on the effect of network parameters on the resulting activity patterns. We used a set of neuronal parameters that adequately reproduced the dynamics of cortical neurons (Izhikevich 2003). The applied set contained a mixture of all neuronal cell types that exist in the cortex, which gave our simulations a certain degree of robustness against variations 
Fig. 9 a An example of NB profile set acquired from a 'pacemaker-driven' NN model with adaptive synapses,

$K_{\max }=1000, D_{\max }=15 \mathrm{~ms}$,

$R=80$ and $4 \%$ of pacemakers

with stronger synaptic

couplings. Black dashed line corresponds to the median and black solid lines are the percentiles. Inset shows the difference between simulated (red lines) and experimental (black lines) percentiles and medians acquired from culture \#4 as in Fig. Fig. 5a. b Rs and Fs distributions acquired from 'pacemaker-driven' simulations with adaptive synapses
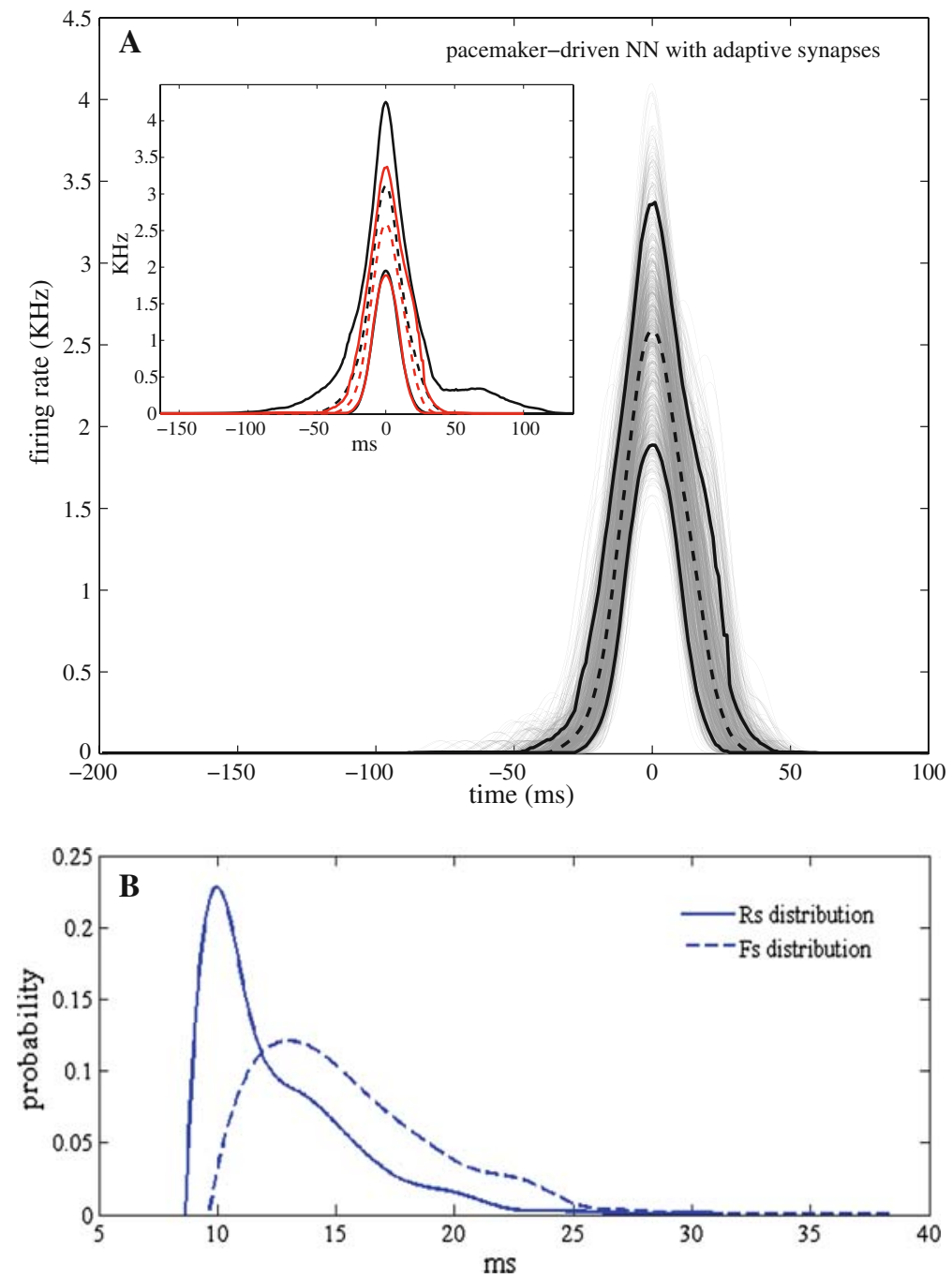

of cell properties. However, it should be noted that our results might be affected by changes in cell properties.

The synaptic parameters for a phenomenological model of STP were estimated for couplings in cortical networks (Markram et al. 1998; Markram 2000).

Our models lack morphologic information which might link model parameters such as connectivity and transmission delay to each other. We feel that these limitations may explain the lack of variability in pre-phases and the complete lack of after-phases in our simulated NB profiles. Wagenaar et al. (2006) clearly showed that the long-tail feature may appear in cultures with about 50,000 (or more) neurons only. So, another reason for the lack of tail phases in our simulated burst profiles might have been the comparatively limited number of 5,000 neurons.

\subsection{Origin and development of spontaneous network bursts}

We studied two possible sources to initiate bursting: synaptic noise versus intrinsically active neurons (pacemakers). First, we ran NN simulations with noisy input to every neuron. Then, we switched to pacemaker neurons with stronger synapses. In both the simulations, bursts appeared beyond a certain threshold of network activity. In this article, we observed the activity patterns of both model types to investigate which one fitted best to the experimental data. We found that two features, the length of the burst onset and the ratio between Rs and Fs, made a clear distinction between the models.

\subsubsection{Networks with 'noise-driven' activity}

First, we analyzed 'noise-driven' activity in networks, with normally distributed parameter values. We showed that synchronized bursting in a collection of interconnected neurons is very common. However, there was no (realistic) combination of network parameters that could adequately reproduce experimental data; bursts always had an unrealistic long onset and Rs usually exceeded Fs.

This longer burst onset may be explained by the slower synchronization in noise-driven models. Here, noisy input 
Fig. 10 Sensitivity curves of $\mathrm{mFr}(\mathbf{a})$ and $\mathrm{Rs}(\mathbf{b})$ to connectivity $\left(K_{\max }\right)$, transmission delay $\left(D_{\max }\right)$, and excitatory fraction $(R)$ in 'pacemaker-driven' simulations with adaptive synapses. Gray background areas correspond to experimental mean $\pm \mathrm{SD}$. Different line types and colors correspond to curves simulated with different $R$ and $D_{\max }$, respectively
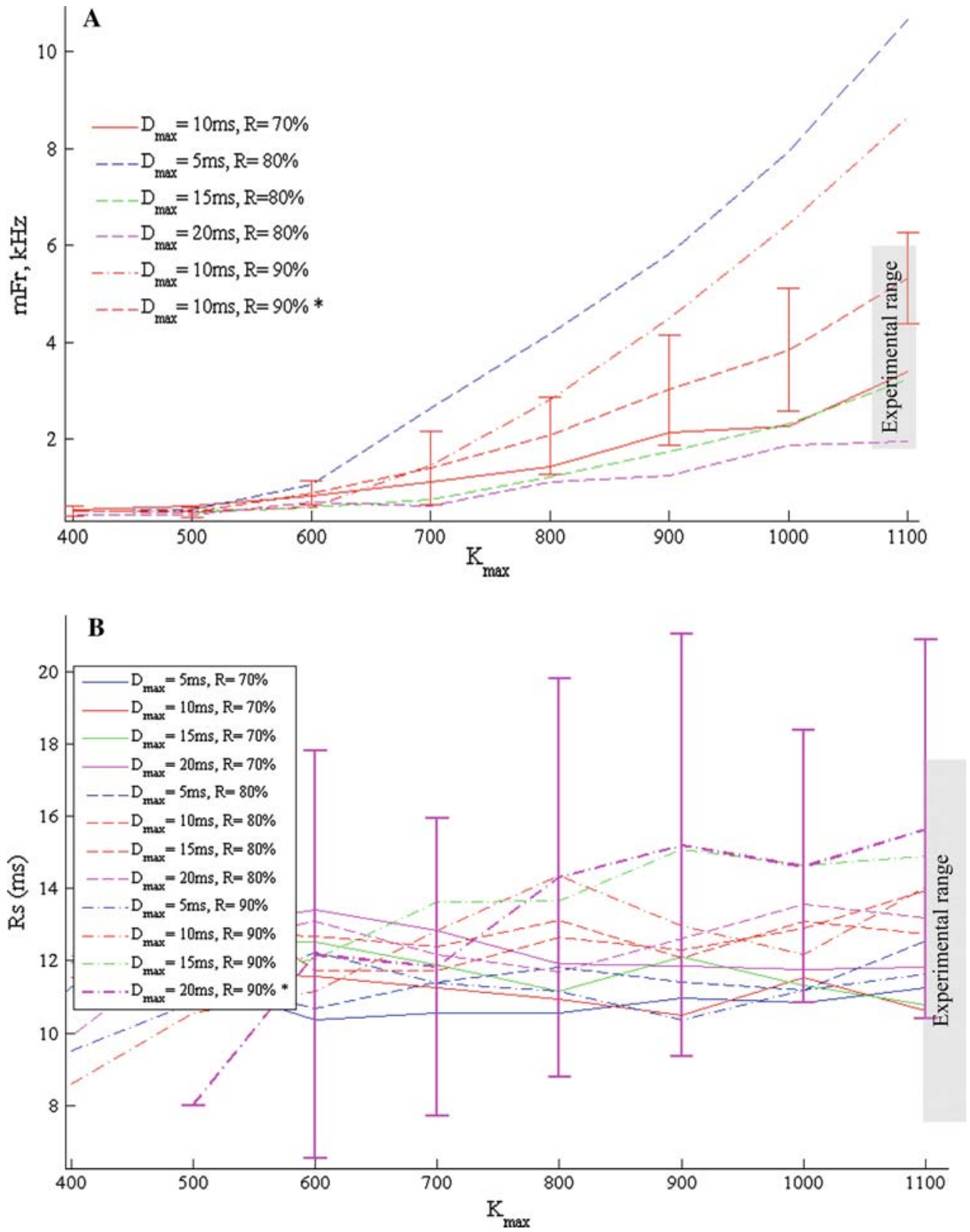

fluctuates around a constant value, whereas in pacemakerdriven models, the input shows much larger variation. During high input, networks may synchronize much faster than during low input. However, when we set noisy input to a level comparable to the maximum pacemaker input, we still found long burst onsets. Probably, a larger fraction of the neurons are in a refractory state when many neurons show irregular spiking because the network continuously receives such high input. Finally, it should be noted that inter burst intervals were much shorter in noise-driven, than in pacemaker-driven simulations. After a network burst, many neurons cannot be excited due to their refractory period, which may largely reduce network excitability. So, if average excitability is lowered the progressively increasing network activity slows down. In our simulations, we mimicked decreased excitability using a lower $R$, which yielded longer burst onsets indeed.

It is hard to find a biological explanation for the $290 \mathrm{~Hz}$ 'threshold'. We showed that all simulated profiles had a long and elevated pre-phase, which started around $100 \mathrm{~ms}$ before the mFr peak. However, $40 \%$ of experimental NBs had no pre-phase at all and in the remaining $60 \%$ pre-phases started much later and usually dropped to $0 \mathrm{kHz}$ before the onset of the main phase (Fig. 5b). As the elevated pre-phase distorted the following rising slope, we decided not to use this model for sensitivity analysis. We also evaluated this model with activity-dependent synaptic plasticity, which did not solve the problem of the long burst onsets. 


\subsubsection{Networks with 'pacemaker-driven' activity}

In order to build a more realistic network model, we added 'pacemaker' neurons with the same or larger synaptic weights than in the rest of the network. A similar approach was used by Wiedemann and Luthi (2003); Vladimirski et al. (2008). In our simulations, the network required a certain amount of 'pacemakers' to produce NBs. First, in simulations where all synaptic weights were normally distributed between 0 and $1 \mathrm{mV}$, the network required $\approx 16 \%$ of 'pacemakers' to produce NBs. 'Pacemaker' neurons fired first and built up a strong wave-like input to the rest of the NN. This input occurred periodically with very low firing activity in between, which allowed the network to fully recover from neuronal and synaptic depression built up during previous NBs. Free from neuronal and synaptic depressions, the NN is more excitable and needs less time to respond to the input. This makes the whole network more sensitive to pacemaker synchronization, which in turn evokes a network-wide burst with relatively short onset. Because pacemaker-driven models with normally distributed synaptic strengths appeared to perform better than noise-driven models, we continued with pacemaker-driven models and did not fully evaluate bimodally distributed synaptic strengths in noise-driven models. However, preliminary results suggest that noise-driven models with bimodally distributed synaptic strengths perform quite similar to pacemaker-driven models without elevated pre-phases and shortened Rs. We also found that NBs are more robust using pacemakers than in the noise-driven case, which is in agreement with the recent theoretical study by Vladimirski et al. (2008).

To investigate how bursts are terminated, we looked at two factors: reduced neuronal excitability after firing and activation of the inhibitory system. In our models, reduced excitability was indicated by a lower average membrane potential ( $v$, see Appendix A). At the burst onset, the total firing rate increased and excitatory feedback raised $v$ of initially silent neurons. This, in turn, recruited new neurons. After firing, $v$ dropped to a relatively low value and recovery was rather slow, which hampered quick reactivation. Thus, bursts progressed as long as the pool of easily activated neurons was large enough to increase network-wide activity. During the burst this pool diminished, until there were not enough easily activated neurons left to maintain synchronized activity, and the burst ceased. Our simulations indeed showed low average $v$ during burst cessation.

Conversely, burst termination might also be caused by activation of the inhibitory system. The example in Fig. 6, shows that the inhibitory system reached its peak firing rate only after the network burst peak. This suggests that the inhibitory system may also play an important role in burst termination, but that it is probably not the sole cause.
Thus, burst cessation, and therefore burst shapes, were affected by both neuronal, as well as network parameters. This implies that our results might be affected by changes in cell properties. However, a profound change of neuronal parameters (setting all excitatory neurons to RS type and all inhibitory neurons to FS type; see Appendix A) yielded only minor changes $(<10 \%)$ of burst shapes. This illustrates the robustness of our model containing a mixture of five fundamental cell types.

Then, the introduction of larger pacemaker synaptic weights allowed us to simulate NB profiles with fewer pacemakers. When the pacemaker synaptic weights ranged from 0 to $3 \mathrm{mV}$, only $4 \%$ of 'pacemakers' in NNs with activityindependent synapses were needed to trigger NBs. All 'pacemaker-driven' simulations showed synchronous activity with a big variety of NB profiles, but they could be divided into two groups: without (40\%) or with $(60 \%)$ a pre-phase, which is similar to experiments. Compared to the noise-driven models, networks with pacemakers produced profiles without elevated and long pre-phases. Furthermore, their main phases were skewed to the left, which yielded shortened Rs.

There are several experimental studies that point at the presence of 'pacemaker-like' neurons in cortical neuronal cultures. Eytan and Marom (2006) called them 'privileged' neurons; Latham et al. (2000b) described them as endogenously active cells, about $32 \%$ of all cells. On average $17 \%$ of spontaneously active neurons can be referred to as burst leaders, as reported by Ham et al. (2008). In our own lab we often find 2 or 3 (out of 60) electrodes with 'pacemakerlike' activity, which is about $4 \%$. We showed that by adding 'pacemakers' to the network, the simulations can reproduce the experimental main phase. Thus, our simulation model helped to relate characteristic NB shapes and the existence of 'privileged' neurons. More experiments, with preferably more electrodes per MEA, are needed to analyze the recruitment of 'pacemaker' neurons and their synchronization.

\subsection{Sensitivity of a network burst profile to network parameters}

The influence of some neuronal and synaptic features on network firing rate was shown in several modeling studies. Fuhrmann et al. (2002) showed that increased spike-adaptation current may explain the emergence of rhythmic fluctuations in the network firing rate. Giugliano et al. (2004) showed that stronger excitatory synapses cause a higher mean firing rate in a bursting network. The stabilizing effect of synaptic depression on network activity was shown by Wang (1999). We noted this latter effect in the network firing rate when adaptive synapses were introduced into the NN model. Synaptic adaptation prevented networks from activity explosions such as shown in Fig. 8a. Moreover, it stabilized Rs 
and Fs means (and SDs) at lower values than in the NNs with activity-independent synapses.

In this study, we focused on the influence of three network parameter ranges on NB features, while neuronal and synaptic parameters were kept in constant ranges (network parameters were changed one after another). We used only network parameters in the physiological range to compare the two models (noise- vs. pacemaker-driven) to experimental recordings.

When STP was introduced most of the simulated $K_{\max }$, $D_{\text {max }}$, and $R$ combinations produced NBs whose profile parameters were in the experimental range. We statistically compared simulated to experimental NB profile parameters $\mathrm{mFr}$, Rs, and Fs, and we analyzed the sensitivity of these parameters to the network parameters $K_{\max }, D_{\max }$, and $R$. In network models with relatively long transmission delays $\left(D_{\max }=[10,15,20] \mathrm{ms}\right)$, higher $K_{\max }$ and $R$ produced NBs with an elevated total firing rate (and $\mathrm{mFr}$ ), which in turn prolonged Rs and Fs. For small $D_{\max }=5 \mathrm{~ms}$, Rs and Fs tended to decrease with higher $K_{\max }$ because it allowed the network to recruit neurons faster. Higher $D_{\max }$ postponed recruitment of neurons in network activity, which made Rs and Fs longer (and the whole NB profile wider).

From this study, we drew two major conclusions. The first is that adaptive synapses stabilize NB parameters in physiologically plausible ranges. Second, we found that in network simulations with normally distributed synaptic strengths the pacemaker-driven models performed better than noise-driven network models.

Open Access This article is distributed under the terms of the Creative Commons Attribution Noncommercial License which permits any noncommercial use, distribution, and reproduction in any medium, provided the original author(s) and source are credited.

\section{Appendix A: Neuron model and parameters}

Izhikevich neuronal model equation has the following form:

$$
\begin{aligned}
v^{\prime} & =0.04 v^{2}+5 v+140-u \\
u^{\prime} & =a(b v-u)
\end{aligned}
$$

Table A1 Synapse parameters

\begin{tabular}{llll}
\hline & $U$ & $\Delta(\mathrm{s})$ & $F(\mathrm{~s})$ \\
\hline$E-E$ & 0.59 & 0.813 & 0 \\
$E-I$ & 0.049 & 0.399 & 1.797 \\
$I-E$ & 0.16 & 0.045 & 0.376 \\
$I-I$ & 0.25 & 0.706 & 0.021 \\
\hline
\end{tabular}

$E$ excitatory, $I$ inhibitory with the auxiliary after-spike resetting

if $v \geq+30 \mathrm{mV}, \quad$ then $\left\{\begin{array}{l}v_{t}=c \\ u_{t}=u_{t-1}+d\end{array}\right.$

Here $v$ represents the membrane potential of the neuron; $u$ denotes a membrane recovery variable, which accounts for the activation of $K^{+}$ionic currents and inactivation of $\mathrm{Na}^{+}$ionic currents or their combination. The parameter $a$ describes the time scale of the recovery variable $u$, so higher values speed up the membrane recovery process. $b$ describes the sensitivity of the recovery variable $u$ to the sub-threshold fluctuations of the membrane potential $v$. Greater values correspond to bigger sub-threshold current influx. The parameters $c$ and $d$ account for action of high-threshold voltage-gated currents activated during the spike, and affect only the after-spike behavior. $c$ describes the after-spike reset value of the membrane potential $v$ caused by the fast high-threshold $K^{+}$conductances. The parameter $d$ denotes after-spike reset of the recovery variable $u$ caused by slow high-threshold $\mathrm{Na}^{+}$and $\mathrm{K}^{+}$conductances and describes the total amount of outward minus inward currents activated during the spike (Izhikevich 2003, 2007). The phase plane analysis show that the equation system (A1) replicates behavior of other detailed models such as FitzHugh-Nagumo, HodgkinHuxley model, etc. in sub-threshold area, and thus, it captures their spiking behavior. Geometrical derivation of this model from the Hodgkin-Huxley equations is provided by Izhikevich (2007). In our simulations, this spiking model mimics the behavior of several types of cortical neurons. Excitatory neurons exhibited regular spiking (RS), intrinsically bursting (IB), and chattering ( $\mathrm{CH})$ behavior; and inhibitory neurons exhibited fast spiking (FS) and low-threshold spiking (LTS) dynamics. These dynamics correspond to the following settings: $\left(a_{i} ; b_{i}\right)=(0.02 ; 0.2),\left(c_{i} ; d_{i}\right)=(-65 ; 8)+$ $(15 ;-6) r_{i} ;$ and $\left(a_{i} ; b_{i}\right)=(0.02 ; 0.25)+(0.08 ;-0.05) r_{i}$, $\left(c_{i} ; d_{i}\right)=(-65 ; 2)$ were assigned to constants of excitatory and inhibitory neurons, respectively, where $r_{i}$ is a random variable normally distributed on the interval $[0,1]$, and $i$ is the neuron index. This choice of $a, b, c$, and $d$ corresponds to a biologically plausible range (Izhikevich 2003).

Most of the biologists agree that these types represent the most fundamental classes of firing patterns observed in the mammalian neocortex. These classes were described by Izhikevich (2007) according to how they responded to injected DC current:

1. RS neurons represent spiny stellate cells in layer 4 and pyramidal cells in layers $2,3,5$, and 6 . They fire spikes with adapting frequency and have type- 1 excitability.

2. IB neurons represent excitatory pyramidal neurons found all over cortical layers. These neurons can fire bursts at the beginning of DC and then switch to the tonic spiking mode. 
3. $\mathrm{CH}$ neurons represent spiny stellate or pyramidal neurons of layers 2-4. They fire regular bursts and are also known as fast rhythmic bursting neurons.

4. FS neurons are the spiny or aspiny nonpyramidal cells. These inter-neurons fire high-frequency spikes with relatively constant period and exhibit type- 2 excitability.

5. LTS neurons represent nonpyramidal inter-neurons. They fire low-threshold spikes with emphasized spike frequency adaptation (Izhikevich 2007).

We modeled the intrinsic activation feature in 'pacemaker' neurons by setting $b$ to values around 0.26 .

\section{Appendix B: Synapse model and parameters}

In our simulation models, we used both activity-independent and activity-dependent synapses. First, we simulated networks with activity-independent synapses only. Then, we changed to activity-dependent synapses and compared the results. Thus, the synaptic weight range was constant in some simulations and in other simulations it varied according to the well-known STP model (Markram et al. 1998; Markram 2000).

The dynamics of both the types of synapse over time are defined by

$\frac{\mathrm{d} I(t)}{\mathrm{d} t}=-\frac{I(t)}{\tau_{\mathrm{syn}}}+W \delta\left(t-t_{\mathrm{sp}}\right)$

where $I$ is the synaptic current, which decays exponentially with time constant $\tau_{\text {syn }}$, except when a spike occurs in the pre-synaptic neuron at time $t_{\text {sp. }}$. The time constant $\tau_{\text {syn }}$ ranged from 3 to $15 \mathrm{~ms}$ throughout different synapses.

Synapse adaptation dynamics were modeled via the following set of equations:

$$
\begin{aligned}
w_{k} & =A \cdot B_{k} \cdot y_{k} \\
y_{k} & =U+y_{k-1} \cdot(1-U) \cdot \exp \left(-\Delta_{k-1} / F\right) \\
B_{k} & =1+\left(B_{k-1}-y_{k-1} \cdot B_{k-1}-1\right) \cdot \exp \left(-\Delta_{k-1} / F\right) \\
y_{1} & =U \\
B_{1} & =1
\end{aligned}
$$

where the notation $w_{k}$ denotes one synaptic weight from $W$ for the $k$ th spike, $y$ is the running variable for synaptic utilization, and $B$ is the running variable for synaptic availability, with $y$ and $B=[0,1]$. The constants $U, \Delta$, and $F$ represent the release probability for the first spike, the depression time constant, and facilitation time constant, respectively. We used experimental data for $U, \Delta$, and $F$ parameters from Markram et al. (1998); Markram (2000) for all four synapse types, see Table A1. Markram et al. showed that these settings lead to biologically plausible synapse models.

\section{References}

Amari S-I (1972) Characteristics of random nets of analog neuron-like elements. IEEE Trans Sys Man Cybern SMC 2:643657

Brunel N, Hakim V (1999) Fast global oscillations in networks of integrate-and-fire neurons with low firing rates. Neural Comput 11:1621-1671

Brunel N, Wang XJ (2003) What determines the frequency of fast network oscillations with irregular neural discharges? I. Synaptic dynamics and excitation-inhibition balance. J Neurophysiol 90:415-430

Destexhe A, Badoual M, Piwkowska Z, Bal T, Rudolph M (2004) A novel method for characterizing synaptic noise in cortical neurons. Neurocomputing 58-60:191-196

Eytan D, Marom S (2006) Dynamics and effective topology underlying synchronization in networks of cortical neurons. J Neurosci 26:8465-8476

Feinerman O, Segal M, Moses E (2007) Identification and dynamics of spontaneous burst initiation zones in unidimensional neuronal cultures. J Neurophysiol 97:2937-2948

French DA, Gruenstein EI (2006) An integrate-and-fire model for synchronized bursting in a network of cultured cortical neurons. J Comput Neurosci 21:227-241

Fuhrmann G, Markram H, Tsodyks M (2002) Spike frequency adaptation and neocortical rhythms. J Neurophysiol 88:761-770

Gibson JR, Connors BW (2003) Chemical and electrical synapses in neocortex. In: Arbib MA (ed) Handbook of brain theory and neural networks. 2. MIT Press, Cambridge, MA, London, pp 725-729

Giugliano M, Darbon P, Arsiero M, Lüscher HR, Streit J (2004) Single-neuron discharge properties and network activity in dissociated cultures of neocortex. J Neurophysiol 92:977-996

Ham MI, Bettencourt LM, McDaniel FD, Gross GW (2008) Spontaneous coordinated activity in cultured networks: analysis of multiple ignition sites, primary circuits, and burst phase delay distributions. J Comput Neurosci 24:346-357

Hansel D, Sompolinsky H (1996) Chaos and synchrony in a model of a hypercolumn in visual cortex. J Comput Neurosci 3:7-34

Hubbard JI, Stenhouse D, Eccles RM (1967) Origin of synaptic noise. Science 157:330-331

Ichikawa M, Muramoto K, Kobayashi K, Kawahara M, Kuroda Y (1993) Formation and maturation of synapses in primary cultures of rat cerebral cortical cells: An electron microscopic study. Neurosci Res 16:95-103

Izhikevich EM (2003) Simple model of spiking neurons. IEEE Trans Neural Netw 14:1569-1572

Izhikevich EM (2007) Dynamical systems in neuroscience: the geometry of excitability and bursting. The MIT Press, London

Jimbo Y, Kawana A, Parodi P, Torre V (2000) The dynamics of a neuronal culture of dissociated cortical neurons of neonatal rats. Biol Cybern 83:1-20

Kamioka H, Maeda E, Jimbo Y, Robinson HPC, Kawana A (1996) Spontaneous periodic synchronized bursting during formation of mature patterns of connections in cortical cultures. Neurosci Lett 206:109-112

Latham PE, Richmond BJ, Nirenberg S, Nelson PG (2000a) Intrinsic dynamics in neuronal networks. II. Experiment. J Neurophysiol $83: 828-835$

Latham PE, Richmond BJ, Nelson PG, Nirenberg S (2000b) Intrinsic dynamics in neuronal networks. I. Theory. J Neurophysiol 83:808827

Le Feber J, Rutten WLC, Stegenga J, Wolters PS, Ramakers GJA, Van Pelt J (2007) Conditional firing probabilities in cultured neuronal networks: a stable underlying structure in widely varying spontaneous activity patterns. J Neural Eng 4:54-67 
Magee JC, Cook EP (2000) Somatic EPSP amplitude is independent of synapse location in hippocampal pyramidal neurons. Nat Neurosci 3:895-903

Markram H (2000) Organizing principles for a diversity of GABAergic interneurons and synapses in the neocortex. Science 287: $273-278$

Markram H, Wang Y, Tsodyks M (1998) Differential signaling via the same axon of neocortical pyramidal neurons. Proc Natl Acad Sci USA 95:5323-5328

Mehring C, Hehl U, Kubo M, Diesmann M, Aertsen A (2003) Activity dynamics and propagation of synchronous spiking in locally connected random networks. Biol Cybern 88:395-408

Muller TH, Swandulla D, Zeilhofer HU (1997) Synaptic connectivity in cultured hypothalamic neuronal networks. J Neurophysiol 77:3218-3225

Muramoto K, Ichikawa M, Kawahara M, Kobayashi K, Kuroda Y (1993) Frequency of synchronous oscillations of neuronal activity increases during development and is correlated to the number of synapses in cultured cortical neuron networks. Neurosci Lett 163:163-165

Nesse WH, Borisyuk A, Bressloff PC (2008) Fluctuation-driven rhythmogenesis in an excitatory neuronal network with slow adaptation. J Comput Neurosci 25:317-333

Persi E, Horn D, Volman V, Segev R, Ben-Jacob E (2004) Modeling of synchronized bursting events: the importance of inhomogeneity. Neural Comput 16:2577-2595

Stegenga J, Le Feber J, Marani E, Rutten WLC (2008) Analysis of cultured neuronal networks using intra-burst firing characteristics. IEEE Trans Biomed Eng 55(4):1382-1390

Swadlow HA, Waxman SG (1975) Observations on impulse conduction along central axons. Proc Natl Acad Sci USA 72:5156-5159

Toledo-Rodriguez M, Gupta A, Wang Y, Wu CZ, Markram H (2003) Neocortex: basic neuron types. In: Arbib MA (ed) The handbook of brain theory and neural networks. 2. MIT Press, Cambridge, MA, London, pp 719-725

Tsodyks M, Pawelzik K, Markram H (1998) Neural networks with dynamic synapses. Neural Comput 10:821-835
Tsodyks M, Uziel A, Markram H (2000) Synchrony generation in recurrent networks with frequency-dependent synapses. J Neurosci RC50:1-5

Van Habets AMMC, Van Dongen AMJ, Huizen F, Corner MA (1987) Spontaneous neuronal firing patterns in fetal rat cortical networks during development in vitro: a quantitative analysis. Exp Brain Res 69:43-52

Van Huizen F, Romijn HJ, Habets AMMC (1985) Synaptogenesis in rat cerebral cortex cultures is affected during chronic blockade of spontaneous bioelectric activity by tetrodotoxin. Develop Brain Res 19:67-80

Van Pelt J, Wolters PS, Corner MA, Rutten WLC, Ramakers GJA (2004a) Long-term characterization of firing dynamics of spontaneous bursts in cultured neural networks. IEEE Trans Biomed Eng 51:2051-2062

Van Pelt J, Corner MA, Wolters PS, Rutten WLC, Ramakers GJA (2004b) Longterm stability and developmental changes in spontaneous network burst firing patterns in dissociated rat cerebral cortex cell cultures on multielectrode arrays. Neurosci Lett 361:86-89

Van Vreeswijk C, Hansel D (2001) Patterns of synchrony in neural networks with spike adaptation. Neural Comput 13:959-992

Van Vreeswijk C, Abbott LF, Ermentrout GB (1994) When inhibition not excitation synchronizes neural firing. J Comput Neurosci $1: 313-321$

Vladimirski BB, Tabak J, O’Donovan MJ, Rinzel J (2008) Episodic activity in a heterogeneous excitatory network, from spiking neurons to mean field. J Comput Neurosci 25:39-63

Wagenaar DA, Pine J, Potter SM (2006) An extremely rich repertoire of bursting patterns during the development of cortical cultures. BMC Neurosci 11:1-18

Wang XJ (1999) Synaptic basis of cortical persistent activity: the importance of NMDA receptors to working memory. J Neurosci 19:9587-9603

Wiedemann UA, Luthi A (2003) Timing of network synchronization by refractory mechanisms. J Neurophysiol 90:3902-3911 